\title{
Astex's journey of fragment-based drug discovery using cryo-EM
}

\author{
M Saur ${ }^{1}$ \\ ${ }^{I}$ Astex Pharmaceuticals, Cambridge, UK \\ michael.saur@astx.com
}

Recent advances in electron cryo-microscopy (cryo-EM) structure determination have pushed the resolutions obtainable by the method into the range widely considered to be of utility for drug discovery. In light of these advances, Astex has decided to heavily invest in this technology and has developed a streamlined pipeline to facilitate fragment-based drug discovery (FBDD) using cryo-EM. This presentation will give an overview of the cryo-EM journey which Astex has embarked upon since 2016. It will also show in-house results to demonstrate that the current reproducibility, quality, and throughput of cryo-EM are compatible with FBDD (see also Saur, Hartshorn et al. (2020) Drug Discovery Today).

Acta Cryst. (2020). A76, a62 